\title{
Optical bright and dark states in side-coupled resonator structures
}

\author{
Philip Chak, Joyce K. S. Poon,* and Amnon Yariv \\ Department of Applied Physics and Department of Electrical Engineering, California Institute of Technology, \\ 1200 East California Boulevard, MC 128-95, Pasadena, California 91125, USA \\ *Corresponding author: poon@caltech.edu
}

Received May 3, 2007; revised May 3, 2007; accepted May 4, 2007; posted May 7, 2007 (Doc. ID 77788); published June 18, 2007

We analyze side-coupled standing-wave cavity structures consisting of Fabry-Perot and photonic crystal resonators coupled to two waveguides. We show that optical bright and dark states, analogous to those observed in coherent light-matter interactions, can exist in these systems. These structures may be useful for variable, switchable delay lines. (0) 2007 Optical Society of America

OCIS codes: $230.5750,230.7370$.

In this Letter, we show the unique dispersion properties of single-mode, side-coupled (SC) cavity structures can be used to generate large variable, switchable delays. Using a transfer-matrix method, we first calculate the dispersion and transmission properties of SC structures based on coupled Fabry-Perot (FP) resonators. We show these structures support "bright" and "dark" states, accessible by tuning the relative phase of the input fields. In analogy with electromagnetically induced transparency (EIT) [1], here a dark state is one in which the resonators are not excited by the optical signal even on resonance. We then show that these bright and dark states also exist in photonic crystal waveguide-cavity structures. Finally, we illustrate how these dark and bright states can be used to construct variable, switchable delay lines.

We first consider structures consisting of two waveguides side coupled to a periodic chain of FP resonators. They can take the form of either (i) a FP coupled-resonator optical waveguide (CROW) side coupled to two waveguides (FP-SC-CROW) as in Fig. 1(a) or (ii) a SC integrated spaced sequence of resonators (SCISSOR) with FP cavities (FP-SCISSOR) as shown in Fig. 1(b) [2,3]. The primary difference between the devices in Figs. 1(a) and 1(b) is the degree of coupling between adjacent cavities: the FP-SCCROW reduces to the FP-SCISSOR in the limit of zero interresonator coupling. In general, the FP resonators in the middle waveguide can be replaced with other single-mode cavities, as long as the resonators support only one mode on resonance. In contrast, microrings, for example, support two degenerate modes on resonance.

To analyze the coupled waveguide structures in Fig. 1, we employ a transfer-matrix method in which we propagate the incoming and outgoing fields along $z$. In general, the transmission properties of each unit cell in Fig. 1 can be described by a $6 \times 6$ matrix; the field at a particular point in $z$ can be described by $\left[\begin{array}{llll}e_{1} & e_{2} & \cdots & e_{6}\end{array}\right]^{T}$. The vector includes both the forward- and backward-propagating fields in the resonator and the two waveguides [4]. To relate the fields at $z=0$ and $z=\Lambda$ for a structure that has inver- sion symmetry with respect to the center of the unit cell, we use the scattering matrix

$$
\left[\begin{array}{l}
\mathbf{e}_{+}^{\prime} \\
\mathbf{e}_{-}
\end{array}\right]=\left[\begin{array}{ll}
\mathbf{T} & \mathbf{R} \\
\mathbf{R} & \mathbf{T}
\end{array}\right]\left[\begin{array}{l}
\mathbf{e}_{+} \\
\mathbf{e}_{-}^{\prime}
\end{array}\right],
$$

where $\mathbf{e}_{+}=\left[\begin{array}{lll}e_{1} & e_{3} & e_{5}\end{array}\right]^{T}$ and $\mathbf{e}_{-}=\left[\begin{array}{lll}e_{2} & e_{4} & e_{6}\end{array}\right]^{T}$. $\mathbf{T}$ and $\mathbf{R}$ are $3 \times 3$ matrices describing the transmission and reflection properties of the unit cell, and their explicit forms are presented in [4]. The scattering matrices can be made more tractable by accounting for nearest waveguide coupling only and neglecting the coupling between the outer waveguides and the gratings. Including the effect of more general coupling between waveguides and cavities in the grating regions will lead to quantitative, but not qualitative, changes in our results. Casting Eq. (1) in the form of a transfer matrix, we have

$$
\left[\begin{array}{l}
\mathbf{e}_{+}^{\prime} \\
\mathbf{e}_{-}^{\prime}
\end{array}\right]=\mathbf{M}\left[\begin{array}{l}
\mathbf{e}_{+} \\
\mathbf{e}_{-}
\end{array}\right] \equiv\left[\begin{array}{cc}
\mathbf{T}-\mathbf{R T}^{-1} \mathbf{R} & \mathbf{R T}^{-1} \\
-\mathbf{T}^{-1} \mathbf{R} & \mathbf{T}^{-1}
\end{array}\right]\left[\begin{array}{l}
\mathbf{e}_{+} \\
\mathbf{e}_{-}
\end{array}\right],
$$

with the transmission properties of our structures described by the $6 \times 6$ transfer matrix $\mathbf{M}$.

To determine the dispersion relation of the infinite, periodic structure, we apply Bloch's theorem [5] and require $\operatorname{det}\left|\mathbf{M}-\mathbf{I} e^{i K \Lambda}\right|=1$, where $\mathbf{I}$ is the identity matrix, $K$ is the Bloch wavenumber, and $\Lambda$ is the period of the structure along $z$. For each frequency of interest, we numerically diagonalize $\mathbf{M}$ and then solve for the corresponding Bloch wavenumber. In the case of
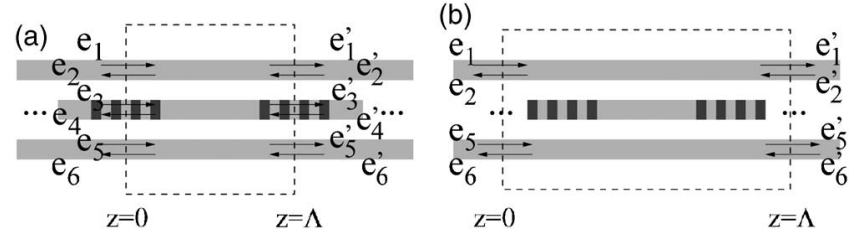

Fig. 1. Schematic of standing-wave resonators side coupled to two waveguides. The light gray regions represent the waveguiding sections and the dark regions represent the high-index regions in the reflectors. (a) FP-SCCROW structure. (b) Two-channel FP-SCISSOR structure. 
an FP-SCISSOR, we are primarily interested in modes that decay to zero deep inside the gratings, consistent with the assumption that there is no interresonator coupling. Therefore, to determine the dispersion relation, we collapse the $6 \times 6$ matrix to a 4 $\times 4$ matrix by only accounting for $\left[\begin{array}{llll}e_{1} & e_{2} & e_{5} & e_{6}\end{array}\right]^{T}$. This is equivalent to assuming that the gratings are perfectly reflecting and that outgoing light from the cavities in the grating regions is a source of loss in the structure. In the numerical examples that follow, the FP-SCISSOR structures are designed with a high mirror reflectivity such that the perfectly reflecting assumption is valid.

The dispersion relations of the structures in Fig. 1 are depicted in Figs. 2 and 3. For both the FP-SCCROW and FP-SCISSOR calculations, the gratings consist of alternating layers satisfying the quarterwavelength condition at $1.55 \mu \mathrm{m}$ for indices of 3.25 and 2.25. The cavities are $12 \mu \mathrm{m}$ long with an index of 3.25 and a waveguide coupling strength of 8.3 $\times 10^{-3} \mu \mathrm{m}^{-1}$. For the FP-SC-CROW, ten grating periods separate successive cavities. For the FPSCISSOR, 100 grating periods separate successive cavities. In the figures, only the dispersion relations of nonevanescent Bloch modes with real wavenumbers are shown. The eigenmodes of the structures can be understood in terms of two different sets of modes: the dark modes that have a constant group velocity and the bright modes. The dark modes propagate unaffected by the cavities (and hence the name "dark") at the phase velocity of the waveguides and are represented by the linear dispersion curves. In contrast, microring SCISSORs and CROWs commonly analyzed in the literature do not support the dark modes $[2,6]$.

For the FP-SC-CROW, the bright-mode dispersion relations in Fig. 2(a) can be understood in terms of the coupling between the CROW modes and the waveguide modes. The band labeled "CROW-like band" in Fig. 2(a) reduces to the CROW dispersion relation when the waveguide-resonator coupling is zero [Fig. 2(b)]. The anticrossing of the waveguidedispersion relations and the $\mathrm{CROW}$ band modifies the CROW band near $K \Lambda / \pi=0.8$ and leads to the formation of a bandgap for the bright modes. Because of
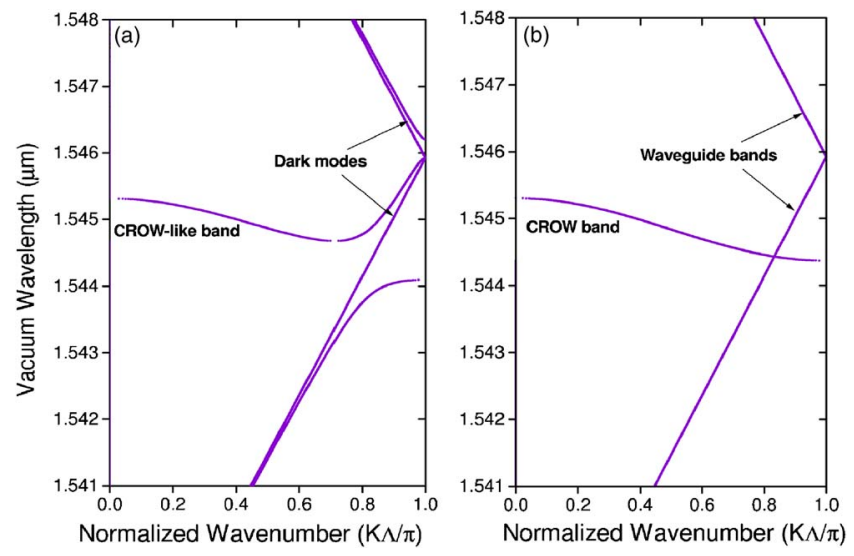

Fig. 2. (Color online) Dispersion relation of an FP-SCCROW in the limit of (a) weak waveguide-cavity coupling and (b) no waveguide-cavity coupling.
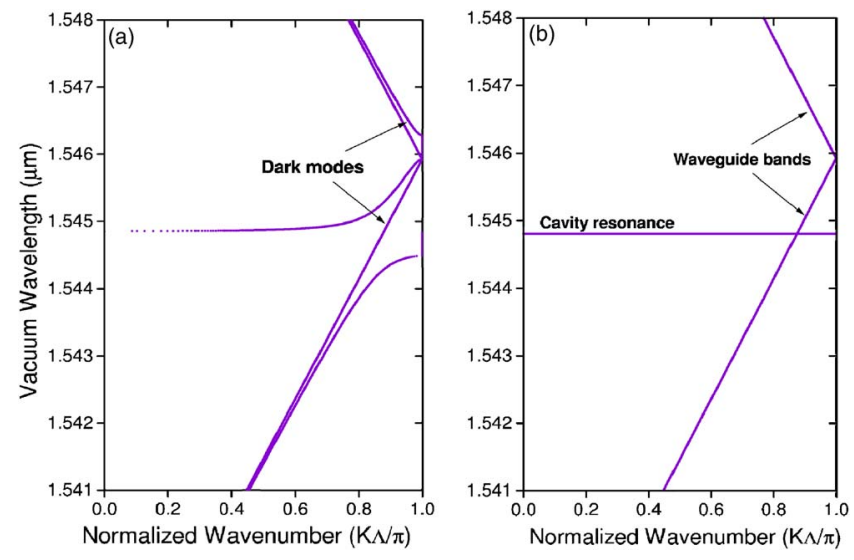

Fig. 3. (Color online) Dispersion relation of an FPSCISSOR in the limit of (a) weak waveguide-cavity coupling and (b) no waveguide-cavity coupling.

the anticrossing, the group velocity can be zero even away from the Brillouin zone edges.

For the FP-SCISSOR, the bright-mode dispersion relation in Fig. 3(a) is qualitatively similar to that of a two-channel microring SCISSOR structure studied in [6], and the physical interpretation of the origins of the bandgaps are the same. One bandgap for the bright mode is centered at the cavity resonance frequency, since resonant wavelengths are reflected by the resonators. The other bandgap arises from the Bragg reflection resultant from the distributed feedback between the resonators and is dependent on the period.

For the dark modes in both structures, the resonators are not excited. This effect can be understood in a similar manner as dark states in EIT and gratings $[7,8]$. In the dark modes, the light in the top and bottom waveguides destructively interfere such that no light is coupled into the resonator, which thus remains dark. The eigenmodes from Eq. (2) reveal that the light in the top and bottom channels are in phase for the bright modes but $\pi$ out of phase for the dark modes. In both cases, the light in the top and bottom waveguides is equal in amplitude.

To illustrate this effect more clearly, we can adopt a simple mode-coupling model of the two (identical) waveguides and the resonators. Let $A, B, C$ represent the envelopes for the forward-going component of the wave in the top waveguide, the FP resonator, and the bottom waveguide, respectively. We can write the coupled-mode equations as

$$
\frac{\mathrm{d} A}{\mathrm{~d} z}=-i \kappa B, \quad \frac{\mathrm{d} B}{\mathrm{~d} z}=-i \kappa A-i \kappa C, \quad \frac{\mathrm{d} C}{\mathrm{~d} z}=-i \kappa B,
$$

where $\kappa$ is the per length coupling coefficient describing the waveguide-cavity coupling. The propagation constants of the envelopes described by Eq. (3) are $\Delta \beta=0, \pm \sqrt{2} \kappa$. The solution $\Delta \beta=0$ implies that the field envelopes are independent of $z$ and $\kappa$. Its corresponding eigenvector is $\left[\begin{array}{lll}A & B & C\end{array}\right]^{T}$ $=1 / \sqrt{2}\left[\begin{array}{ccc}1 & 0 & -1\end{array}\right]^{T}$, precisely representing the dark mode in which no light propagates in the resonators because of destructive interference. Note that since 


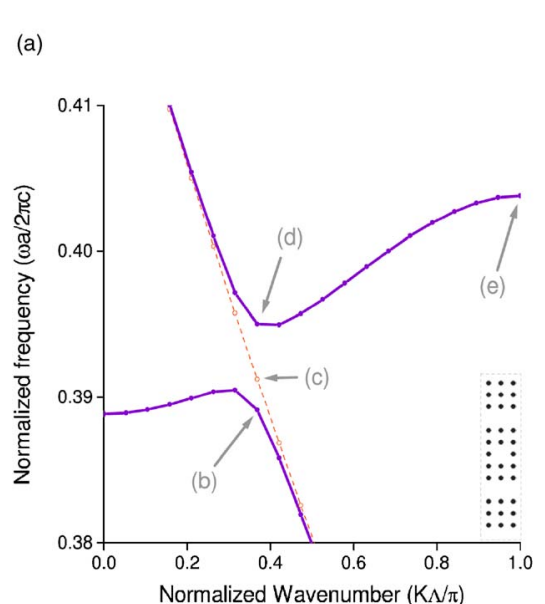

Fig. 4. (Color online) (a) TM dispersion relation of a PCSC-CROW near the resonance frequency of the PC cavity. Inset, unit cell of the structure. The dark rods have $n=3.0$ and $r=0.2 a$, where $a$ is the lattice constant for the square lattice. The background material has $n=1.0$. (b)-(e) Fields labeled in (a).

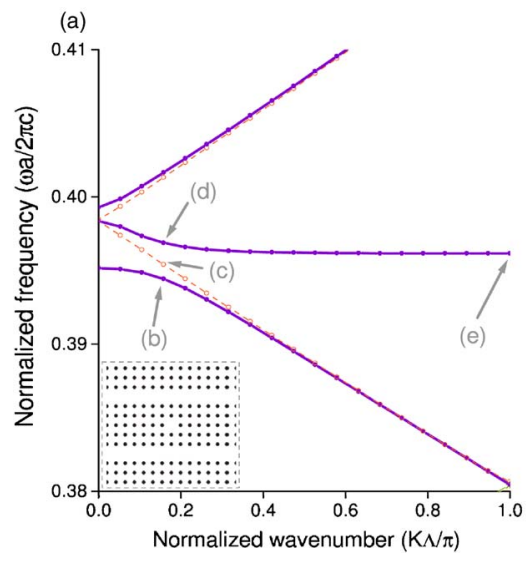

(b)

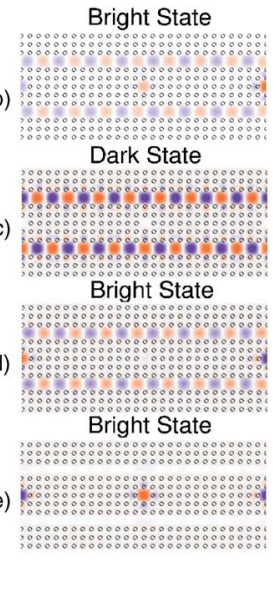

Fig. 5. (Color online) (a) TM dispersion of a PC-SCISSOR. The PC is identical to Fig. 4. Inset, unit cell of the structure. (b)-(e) Fields labeled in (a).

$A=-C$ and $\mathrm{d} B / \mathrm{d} z=0$, no net coupling takes place in the resonators.

Dark and bright states also exist in more general photonic crystal (PC) structures. Insets in Figs. 4(a) and 5(a) show the PC-SC-CROW and PC-SCISSOR. The waveguides and cavities of the structures are formed by line and point defects, respectively. Dispersion relations in close vicinity of the normalized resonance frequency, $\widetilde{\omega}=0.396$, is shown in Figs. 4 and 5 (a), and the corresponding field distributions are depicted in Figs. 4 and 5(b)-5(e). The calculated fields show that the PC structures can exhibit the same characteristics as the FP-SC-CROW and FPSCISSOR structures considered in Figs. 2 and 3.

The bright and dark modes can be used to generate slow light with a variable, switchable delay. Since these modes are distinguishable by the relative phase between the top and bottom waveguides, the delay through these devices can be adjusted by altering the relative phase of the two input beams. When

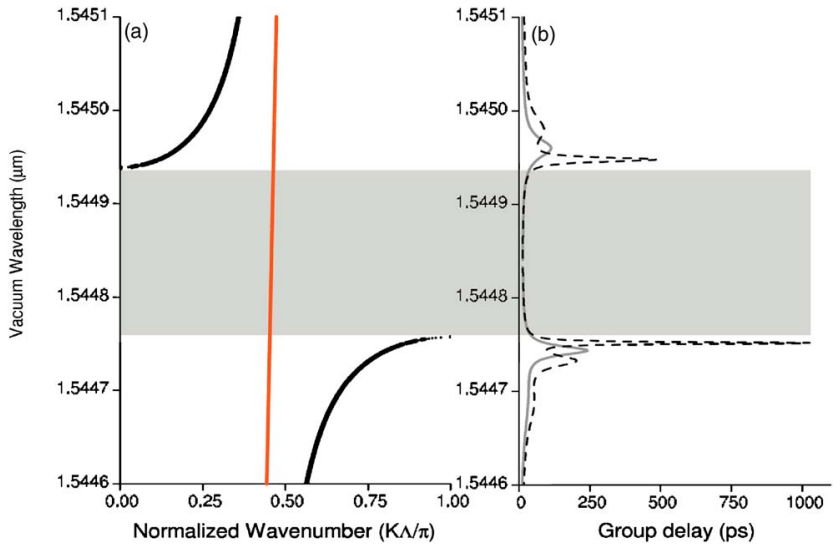

Fig. 6. (Color online) (a) Dispersion relation of an FPSCISSOR with similar parameters as Fig. 3 but with 40 grating periods separating successive cavities. (b) Group delay in finite FP-SCISSORs consisting of five (solid curve) and eight (dashed curve) cavities.

the relative phase between the input beams equal $0(\pi)$, then the bright (dark) state is excited. The group delay in finite FP-SCISSOR structures with five and eight cavities, calculated using a scattering matrix approach based on Eq. (1), is shown in Fig. 6(b). For FP-SCISSORs, at each frequency of interest, one forward-propagating bright (dark) mode is supported. The group delay for the bright modes increases around the band edges. For the dark modes, the group delay (not shown) is approximately 1.3 and $2 \mathrm{ps}$. By switching the relative phase between the input beams, the delay can be changed by 2 orders of magnitude.

In conclusion, we have shown that side-coupled single-mode FP and PC resonator chains support slow-moving bright and fast-moving dark modes. In contrast to the typical scheme of varying the resonator coupling coefficients, the optical delay in these structures can be changed by the phase of the input light.

This work was supported by the Defense Advanced Research Projects Agency (Slow Light Project). P. Chak and J. Poon are grateful for the financial support from the Natural Sciences and Engineering Research Council of Canada.

\section{References}

1. L. V. Hau, S. E. Harris, Z. Dutton, and C. H. Behroozi, Nature 397, 594 (1999).

2. A. Yariv, Y. Xu, R. K. Lee, and A. Scherer, Opt. Lett. 24, 711 (1999).

3. J. E. Heebner and R. W. Boyd, J. Mod. Opt. 49, 2629 (2002).

4. J. K. S. Poon, P. Chak, J. M. Choi, and A. Yariv, "Slowing light with Fabry-Perot resonator arrays," (submitted to J. Opt. Soc. Am. B).

5. A. Yariv and P. Yeh, Optical Waves in Crystals: Propagation and Control of Laser Radiation (Wiley, 1984).

6. S. Pereira, P. Chak, and J. E. Sipe, J. Opt. Soc. Am. B 19, 2191 (2002).

7. J. P. Marangos, J. Mod. Opt. 45, 471 (1998).

8. E. Peral and A. Yariv, J. Lightwave Technol. 17, 942 (1999) 